\title{
Vessel extraction from retinal images by using matched filter and first derivative of Gaussian function
}

\author{
S.J. Deshmukh ${ }^{1}$, S.B.Patil ${ }^{2}$ \\ Dept. E\&TC, Sinhgad College of Engineering, Vadgaon ${ }^{1,2}$
}

\begin{abstract}
Accurate detection of blood vessels from retina is an important job in computer aided diagnosis of Diabetic Retinopathy. The Matched filter is simple \& effectively good method for vessel extraction. But Problem with that it recognizes vessels and non-vessels edges so it will detect false vessels. In this paper we introduce new extension of Matched Filter i.e. FDOG to extract the vessels. The MF-FDOG is combination of Matched filter which is zero mean Gaussian Function and First order derivative of Gaussian. The vessels are detected by thresholding the response of Matched Filter and Threshold is adjusted by image response of FDOG. The proposed method is very simple and it reduces false detection of Matched filter and detects all fine vessels which are not detected by matched filter. It gives good competitive results of vessel detection with low complexity.
\end{abstract}

Keyword: Diabetic Retinopathy (DR), Matched filter (MF), First order derivative of Gaussian Function (FDOG).

\section{I.INTRODUCTION}

Diabetic retinopathy is a micro-vascular complication of diabetes, causing abnormalities in the retinal image. Typically there are no detectable symptoms in the early stages, but the number and severity predominantly increase in time. In the following, the progress of the diseases described in detail. The diabetic retinopathy typically begins as small changes in the retinal capillaries. Diabetic retinopathy is regarded as a retinal vasculature disorder that evolves up to a degree in majority of the patients with diabetes mellitus [1]. Although diabetes itself cannot be prevented, but in many cases its blindness can be moderate and curable if the disease is diagnosed as early as possible.

he World Health Organization (WHO) has calculated that, the number of adults with diabetes in the world would increase tremendously: from 135 million in 1995 to 300 million in 2025 [2]. In India, this increase is expected to be greatest, nearly $195 \%$ from 18 million in 1995.So we need to find something better which can our life better The extraction of blood vessels in retinal images is important step in diagnosis and treatment of DR [3-4]. Vessel extraction is basically a form of line detection problem and there are many methods. A most popular method of segmentation is based on filtering methods $[5-8,14]$

Another is mathematical morphology [9-10] by applying mathematical operators. Trace-based methods [11] mapout the global network of blood vessels after edge detection and by tracing center lines of blood vessels. And last is machine learning methods which are divided into two groups supervised and unsupervised. In Supervised methods [3, 12], some prior information is needed to perform blood segmentation. While unsupervised methods $[11,13]$ do the vessel segmentation without any information.

Among various vessel extraction methods, the matched filter [14]is well known due their simplicity \& effectiveness. In MF vessels are detected by using filtering and thresholding but MF have strong response to vessels as well as non-vessels for e.g. edges of bright spots \& Red lesions. Here MF used knowledge that cross section of vessel is Gaussian shape. Gaussian shape is symmetrical to their peak position. This property is used to distinguish symmetric vessel structure from non-symmetric vessel structure so accuracy of vessel extraction is increased.

In this paper we are using matched filter (i.e. zero mean of Gaussian function) \& first order derivative of Gaussian function. For true vessel, MF will show strong response to its peak position \& weak response (i.e. close to zero) for F-DOG. And for non-vessels, MF and FDOG shows strong response to their peak position. By applying MF-FDOG filters to the retinal image, two responses obtained. First is $\mathrm{H}$ by MF. Second is D by FDOG. Vessels are detected by applying threshold $\mathrm{T}$ to $\mathrm{H}$ response. Where $\mathrm{T}$ is adjusted by $\mathrm{D}$ response So by using MF-FDOG accuracy of system is increased and simplicity is preserved.

The remaining of this paper is formed as below. part 2.review of matched filter, 3..proposed MF-FDOG method, 4.experimental results, 5.Conclusion

\section{II .THE MATCHED FILTER}

The matched filter is introduced in[14] is used to extract vessels in retinal images. In that method they used knowledge that cross-section of vessels is similar to Gaussian function so Gaussian shaped filter is used to match vessels in retinal images.

The MF is defined as,

$$
\begin{gathered}
f(x, y)=\frac{1}{\sqrt{2 \Pi s}} \exp \left(-\frac{x^{2}}{2 s^{2}}\right)-m \text { for }|x|<t . s, \\
|y| \leqslant \frac{L}{2}
\end{gathered}
$$


Where $\mathrm{S}$ is the scale of filter.

$m=\int_{-t s}^{t s} \frac{1}{\sqrt{2 \Pi s}} \exp \left(\frac{-x^{2}}{2 s^{2}}\right) \cdot d x / 2 t s$

$\mathrm{m}$ is normalize the mean value of filter to 0 so smooth background can be removed after filtering.

$\mathrm{L}$ is the length of neighborhood pixel along $\mathrm{y}$-axis, $\mathrm{t}$ is constant which is set to $3, \mathrm{~L}$ is depends upon $\mathrm{s}$. whereas $\mathrm{s}$ decreases $L$ is also decreases. In the implementation, $f(x, y)$ can be rotated in different orientation.

MF is simple for vessel extraction but problem with that, it responds to both vessel as well as non-vessel. Fig1 (a)-(b) shows that problem. In fig MF has strong response to both Gaussians function \& Step edge. So by applying threshold we can classify them as vessel or nonvessel

Therefore the goal of this paper finds the threshold which can separate vessel or non-vessel

\section{III. .THE MF-FDOG FILTER}

As see in part 2 , the MF show strong response to both vessel and step function because it match the shape of both function in some percentage. So it is possible to distinguish vessel from non-vessel. But difference is that for vessel it show strong symmetric response and for nonvessel it show asymmetric response.

Here we introduce scheme which distinguish Gaussian vessel structure from non-vessel. The MF is a zero mean Gaussian Filter and first order derivative of Gaussian is given by

$$
\begin{aligned}
& g(x, y)=-\frac{x}{\sqrt{2 \Pi s^{3}}} \exp \left(\frac{-x^{2}}{2 s^{2}}\right) \\
& \quad \text { for }|x|<t . s, \quad|y|<\frac{L}{2}
\end{aligned}
$$

In this scheme MF and FDOG used in combination to detect vessel and non-vessel [17]l. The Gaussian function i.e. vessel shows strong positive response to MF and For FDOG it is anti-symmetric. Non-vessel shows partially positive response to MF and response of FDOG is positive and symmetric. Fig 1 shows this by using artificial signal, Fig 1(a) shows Gaussian function(Vessel) and step edge function(non-vessel) .Fig (b-1) and (b-2) shows MF and its response. .Fig (b-1) and (b-2) shows $\mathrm{MF}$ and its response. Fig (C-1) and (C-2) shows FDOG and its response. Signal response of $\mathrm{MF}$ is denoted by $\mathrm{H}$. Here we apply Threshold $\mathrm{T}$ to $\mathrm{H}$ curve to classify vessel and non-vessel. Then some non-vessel part is wrongly classify as a vessel

The response of FDOG is D curve. Suppose we apply Threshold $\mathrm{T}$ to $\mathrm{D}$ curve then there is also wrong classification.
Wrong classification in D curve present because there is rapid change at Gaussian peak (at 100) and step edge (at 300). So we directly cannot use FDOG response for classification. Then calculate the local mean value of $\mathrm{D}$ curve detonated by $\mathrm{D}_{\mathrm{m}}$. The local mean value is calculated by averaging the neighboring elements. Fig 1(d) shows $D_{m}$ we can see that in peak area of Gaussian there is strong response of $\mathrm{H}$ but corresponding response of $\mathrm{D}_{\mathrm{m}}$ is low. IN contrast, in case of step edge there is strong response of $\mathrm{H}$ but response of $D_{m}$ is very high. From all the waveforms it indicates that $T$ depends upon $\mathrm{D}_{\mathrm{m}}$. If mag of $\mathrm{D}_{\mathrm{m}}$ is low, then it indicate that vessel may present at neighboring pixel so $\mathrm{T}$ is lower to detect the vessel

And if magnitude of $D_{m}$ is high then it indicate that there are some non-vessel part in neighboring pixel so $\mathrm{T}$ is increased to remove non-vessel.

Finally $\mathrm{T}$ is actually applied on response of MF i.e. ( $\mathrm{H}$ curve) but threshold level is adjusted depending upon FDOG local mean response $\mathrm{D}_{\mathrm{m}}$.

The local mean response of $\mathrm{D}$ is calculated by filtering $\mathrm{D}$ with mean filter.

$\mathrm{D}_{\mathrm{m}}=\mathrm{D} * \mathrm{~W}$

Here $\mathrm{W}$ is $\mathrm{w}^{*} \mathrm{~W}$ filter with each element of filter is $\frac{1}{w^{2}}$. After that local mean image is normalized because we want each element is within $[0,1] . \mathrm{D}_{\mathrm{m}}$ is normalize image of $\mathrm{D}_{\mathrm{m}}$. Threshold $\mathrm{T}$ is given by ,

$\mathrm{T}=\left(1+\mathrm{D}_{\mathrm{m}}\right) * \mathrm{~T}_{\mathrm{c}}$.

Here $T_{c}$ is reference threshold and given by

$\mathrm{T}_{\mathrm{c}}=\mathrm{C}^{*} \mathrm{u}_{\mathrm{H}}$

Here $\mathrm{uH}$ means mean value of $\mathrm{H}$ curve and $\mathrm{C}$ is constant which is set between 2 and 3 depending upon requirement. Then apply Ton $\mathrm{H}$ and final value $\mathrm{m}_{\mathrm{H}}$ is obtained by,

$\{m H=1 \quad H(x, y) \geq T(x, y)$

$\left\{\begin{array}{l}m H=0 \quad H(x, y)<T(x . y)\end{array}\right.$

From above equation it is clear that, suppose there are vessel pixel in image then $D_{m}$ is weak so $T_{H}$ is low thus vessel can be easily detected. And if there are non-vessel part in image $D_{m}$ will high and $T_{H}$ is raise so non vessel edges are suppressed.

Consider below fig. 2 to clarify proposed MF-FDOG

Scheme. Fig 2 (a) shows an original image which includes some bright lesions. Fig 2(b) is response of MF. MF has strong response to both vessels and bright lesions. Fig 2(c) is local mean response of FDOG i.e. $D_{m}$. Fig 2(d) shows the vessel extraction result of MF by applying global threshold. In Fig 2(e) is the result of MF-FDOG filter. It

can be clearly 
INTERNATIONAL JOURNAL OF INNOVATIVE RESEARCH IN ELECTRICAL, ELECTRONICS, INSTRUMENTATION AND CONTROL ENGINEERING Vol. 3, Issue 1, January 2015
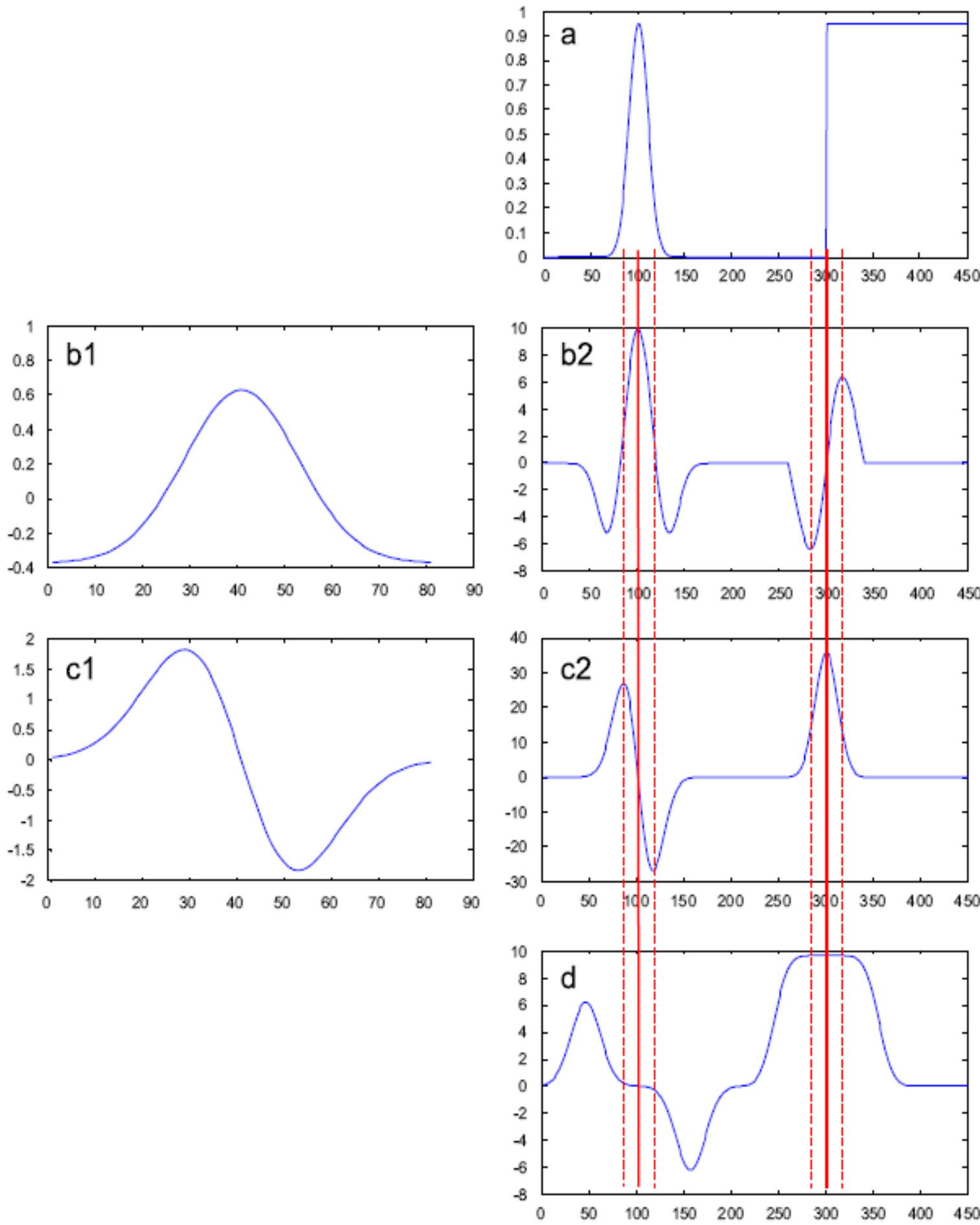

Fig.1.Response of the MF and the FDOG to a Gaussian line cross-section and an ideal step edge: (a) a Gaussian line cross-section and an ideal step edge, (b-1) the MF and (b-2) its filter response, (c-1) the FDOG and (c-2) its filter response and (d) the local mean of the response to the FDOG. 
indicate false detection of bright lesions are greatly true retinal vessel and non-vessels as compared to reduced also many fine vessels are detected which are not conventional MF.

shown in Fig 2(d). So from above, it clearly indicate that proposed MF-FDOG scheme clearly separate out between

a

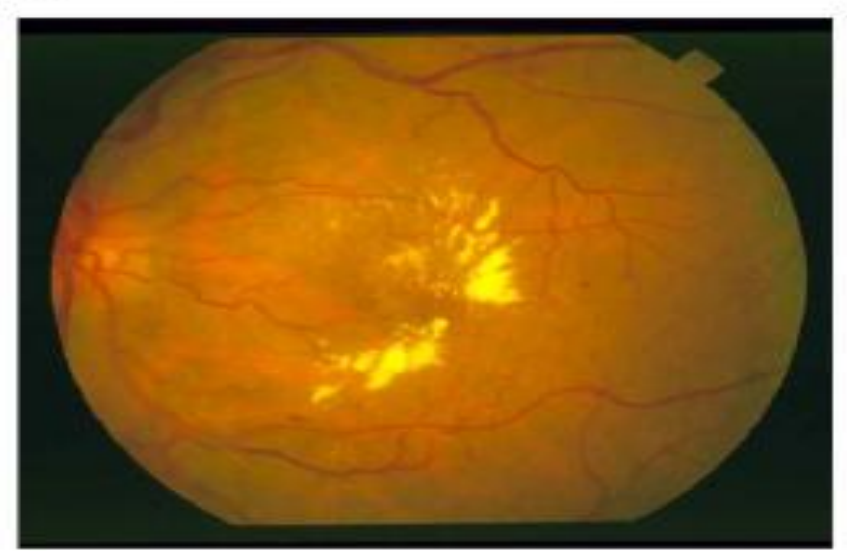

C

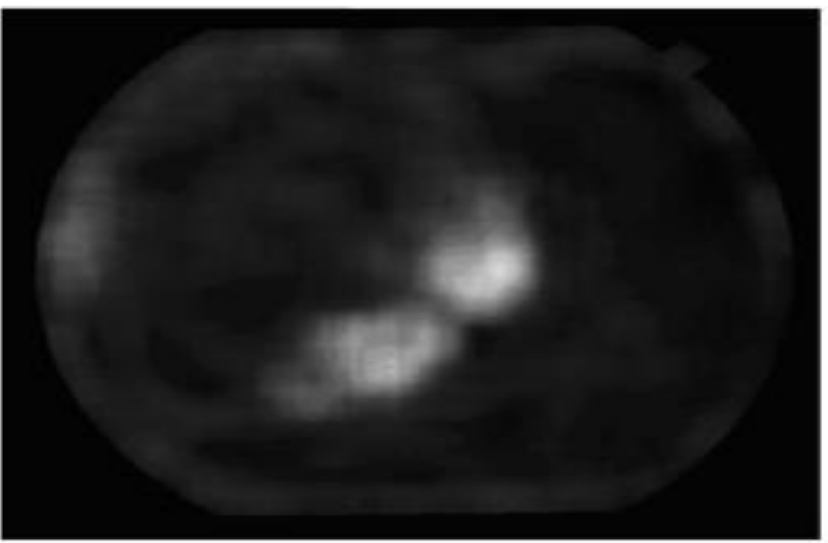

e

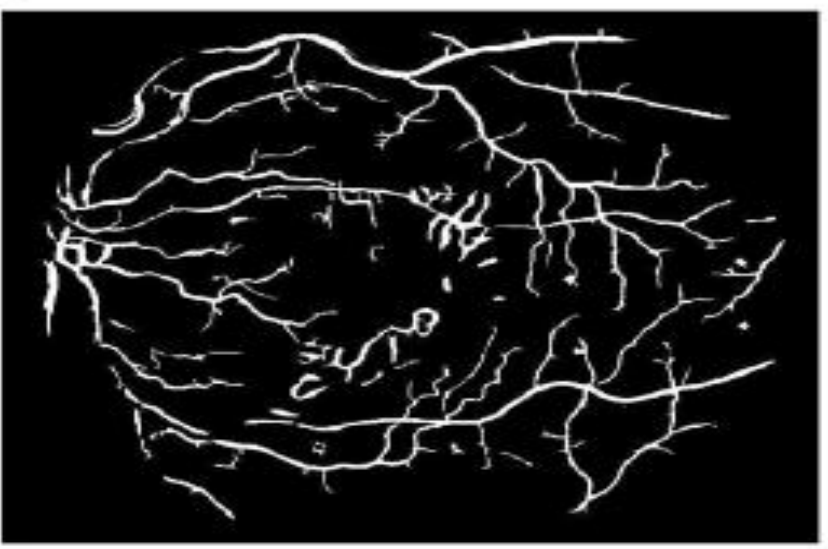

b

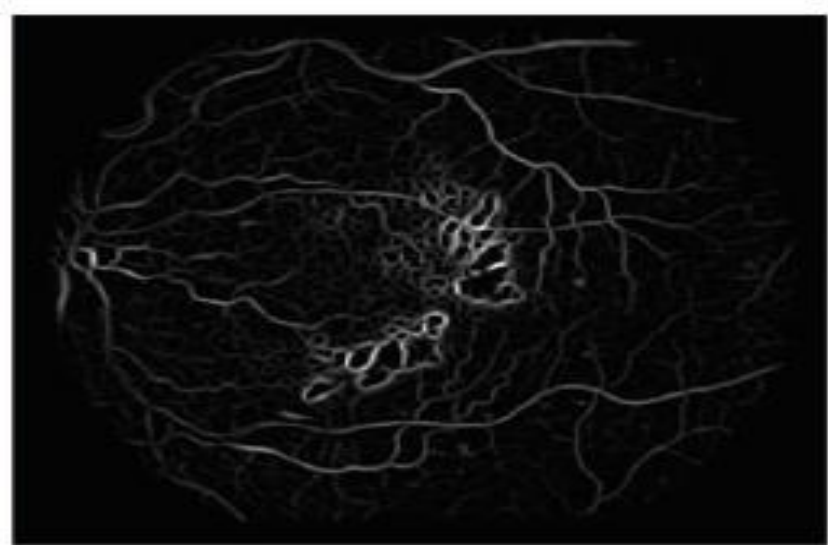

d

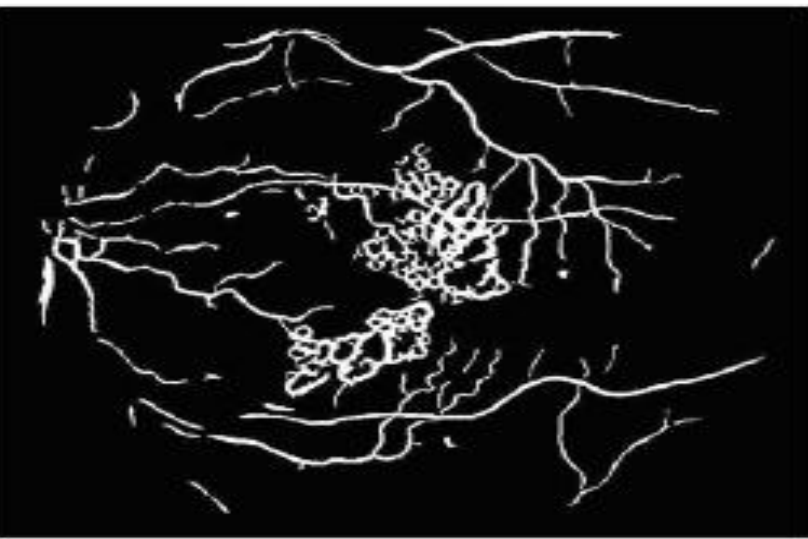

f

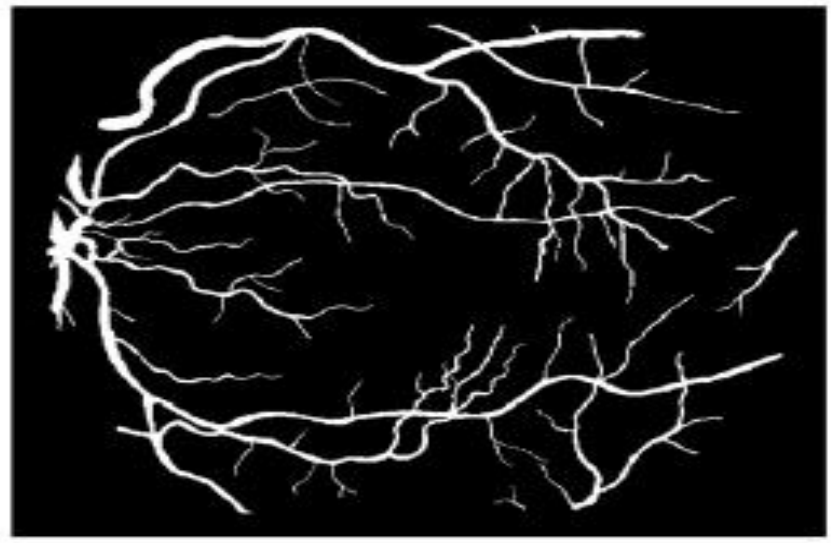

Fig.2.Illustration of the proposed MF-FDOG retinal vessel extraction scheme :(a) the original image,(b)the response map to MF, (c) the local mean of the response to FDOG, (d)the vessel extraction result after applying a global threshold to the MF response map,(e)the extraction result of the proposed scheme and (f)the ground truth vessel map 


\section{IV.EXPERIMENTAL RESULTS}

To extract both thin and thick vessels in image, we apply large scale to thick vessels and small scale to thin vessels. The result of both scales are then combined by using simply OR operation. For wide vessels set $\mathrm{s}=1.5, \mathrm{~L}=9$ and for thin vessels set $\mathrm{s}=1, \mathrm{~L}=5$ in equation (1) and (2). $\mathrm{W}=31 * 31$ (in equation 3 ) and $\mathrm{C}=2.3$ in equation(5) and 8 directions were used in MF-FDOG filtering.

We tested it on DRIVE database .DRIVE database contain 40 images captured by cannon CR5 camera at 45 degree, which digitized as 24 bit gray spatial values of $565 * 584$ pixels. The 40 images are classified as training and test images.

To compare different algorithms of vessel extraction we measure accuracy as performance parameter. Accuracy is total no of correctly classified pixels to total no of pixels inside FOV.The TPR is defined as the ratio of the no of pixels which are correctly classified as vessels to total no of pixels. FPR is the ratio of the non-vessel pixels which is consider as vessels to the total no of non-vessel pixels.

Table 1.Vessel extraction result on DRIVE database

\begin{tabular}{llll} 
Method & TPR & FPR & Accuracy \\
\hline Staal[3] & 0.7194 & 0.0227 & 0.9442 \\
Soares[15] & 0.7283 & 0.0212 & 0.9466 \\
$\begin{array}{l}\text { Mendonca[4] } \\
\text { Matched }\end{array}$ & 0.7344 & 0.0236 & 0.9452 \\
$\begin{array}{l}\text { Filter[5] } \\
\text { Martinez }\end{array}$ & 0.6168 & 0.0259 & 0.9284 \\
perez[16] & 0.7246 & 0.0345 & 0.9344 \\
MF-FDOG & 0.7120 & 0.0276 & 0.9382
\end{tabular}

Table 1 shows result on DRIVE database. The performance measure of Mendonca [4],Matched Filter [5] ,Martinez perez [16] were obtained from their original paper.While Staal[1] and soares[15] are calculated using segmented image from their websites. From above table it indicates that MF-FDOG performs much better than MF but it is slightly inferior compared to other but simple method.

To evaluate the result of MF-FDOG, Consider ROC curve for DRIVE database. Fig 3 shows that for each point we use different threshold to segment the vessels but it give good performance when FP is greater than 0.02 .

\section{ROC Curve of MF-FDOG for DRIVE}

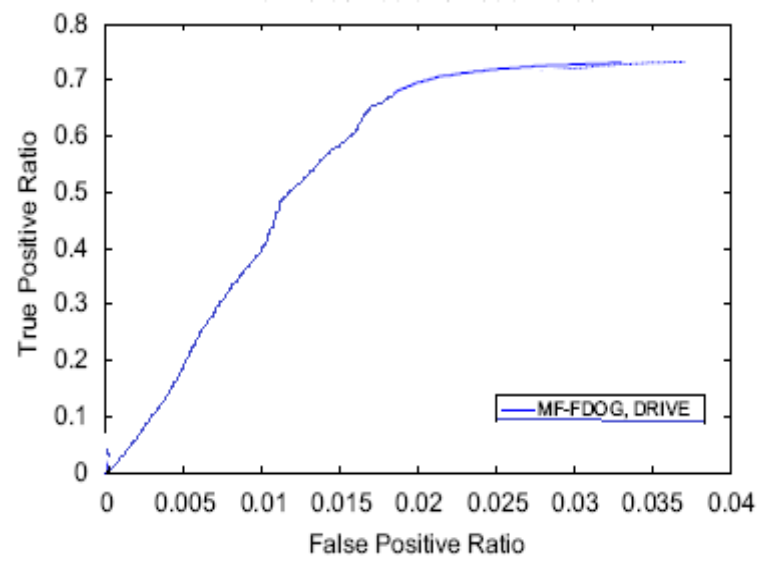

Fig.3. The ROC curve for DRIVE when FP $<0.05$ by the proposed method

\section{V.CONCLUSION}

We propose a vessel extraction method by combining MF and FDOG. The vessels are detected by applying threshold to MF response \& Threshold is calculated by using FDOG. The experimental results clearly indicate that we reduce false detections in MF \& detect all fine vessels. Advantages of this method are that it is simple and easy to implement.

To extract thin and thick vessels we use multiple scales \& combine result by logical OR operation. But resultant vessel map contain some noisy data, this is weak point of the method. So we use some post processing technique to remove noise. Also Sensitivity and accuracy can be improved by handling of branching points and the connectivity to vessels.

\section{REFERENCES}

[1] Jayakumari and T. Santhanam, "Detection of Hard Exudates For Diabetic Retinopathy Using Contextual Clustering And Fuzzy Art Neural Network", Asian Journal of Information Technology, Vol. 6,No.8, pp. 842-846,2007.

[2] R.C.GonzaJez, Digital Image Processing, 2nd ed. PHI, New Delhi110001: Prentice Hall of India, 2006.

[3] M.Niemeijer,J.J.Staal,B.vanGinneken,M.Loog,M.D.Abramoff,Com parativestudy of retinal vessel segmentation methods on a new publicly available database, SPIEMed.Imaging5370(2004)648-656.

[4] A.M.Mendonca, A.Campilho, Segmentation of retinal blood vessels by combining the detection of center lines and morphological reconstruction, IEEE Trans. Med. Imaging25(9)(2006)1200-1213.

[5] M.Cinsdikici, D.Aydin, Detection of blood vessels i n ophthalmoscope images using MF/ant (matched filter/ant colony) algorithm, Compute .Methods and Programs Biomed. 96(2009)85-95.

[6] M.AlRawi, M.Qutaishat, M.Arrar, An improvement matched filter for blood vessel detecetion of digital retinal images,Comput.Biol.Med.37(2007)262-267.

[7] O.Chutatape, L.Zheng, S.Krishnan, Retinal bloodvessel detection and tracking by matched Gaussian and kalman filters, Proc. Eng. Med. Biol. Soc.(1998) 3144-3149.

[8] R.M.Rangayyan, F.J.Ayres, F.Oloumi, F.Oloumi, P.EshghzadehZanjani, Detection of bloodvessels in the retina with multiscale Gabor filters, J. Electron. Imaging17 (2008) 023018.

[9] M.M.Fraz, M.Y.Javed, A.Basit, Evaluation of retinal vessel segmentation methodologies based on combination of vessel center lines and morphologi-Cal processing, in: IEEE International Conference on Emerging Technologies, October 18-19, Rawalpindi, Pakistan, 2008

[10] F.Zana, ,J.C.Klein, Segmentation of vessel-like patterns using mathematical morphology and curvature evaluation, IEEE Trans. Image Process.(2001) 1010-1019.

[11] Y.Tolias, S.Panas, A fuzzy vessel tracking algorithm for retinal images based on fuzzy clustering, IEEE Trans. Med. Imaging (1998) 263-273.

[12] J.J.Staal, M.D.Abramoff, M.Niemeijer, M.A.Viergever, B.vanGinneken, Ridge based vessel segmentation in color images of the retina ,IEEE Trans .Med. Imaging (2004)501-509

[13] S.Garg, J.Sivaswamy, S.Chandra, Unsupervised c urvature - based retinal vessel segmentation, in : Proceedings of the IEEE International Symposiumon Bio-Medical Imaging, 2007, pp. 344-347.

[14] S.Chaudhuri, S.Chatterjee, N.Katz, M.Nelson, M.Goldbaum, Detection of blood vessels in retinal images using two-dimensional matched filters, IEEE Trans. Med. Imaging (1989) 263-269.

[15] J.V.B. Soares, J.J.G. Leandro, R.M. Cesar Jr., H.F. Jelinek, M.J. Cree, Retinal vessel segmentation using the 2-d gabor wavelet and supervised classification, IEEE Trans. Med. Imaging 25 (2006) 1214-1222.

[16] M.E. Martinez-Perez, A.D. Hughes, S.A. Thom, A.A. Bharath, K.H. Parker, Segmentation of blood vessels from red-free and fluorescein retinal images, Med. Image Anal. 11 (1) (2007) 47-61.

[17] Bob Zhang,Lin Zhang,Lei Zhang,Fakhri Karray,'”Retinal vessel extraction by matched filter with First order derivative of Gaussian”@2010 Elseveir Ltd. 